\title{
Structural basis of YAP recognition by TEAD4 in the Hippo pathway
}

\author{
Liming Chen, ${ }^{1,5}$ Siew Wee Chan, ${ }^{1,5}$ XiaoQian Zhang, ${ }^{1}$ Martin Walsh, ${ }^{2}$ Chun Jye Lim, ${ }^{1}$ \\ Wanjin Hong, ${ }^{1,3,7}$ and Haiwei Song ${ }^{1,4,6}$ \\ ${ }^{1}$ The Cancer and Developmental Cell Biology Division, Institute of Molecular and Cell Biology, Proteos, Singapore 138673; \\ ${ }^{2}$ MRC France, CRG BM14, ESRF, BP220, Grenoble, France; ${ }^{3}$ Department of Biochemistry, National University of Singapore, \\ Singapore 117543; ${ }^{4}$ Department of Biological Sciences, National University of Singapore, Singapore 117543
}

The Hippo signaling pathway controls cell growth, proliferation, and apoptosis by regulating the expression of target genes that execute these processes. Acting downstream from this pathway is the YAP transcriptional coactivator, whose biological function is mediated by the conserved TEAD family transcription factors. The interaction of YAP with TEADs is critical to regulate Hippo pathway-responsive genes. Here, we describe the crystal structure of the YAP-interacting C-terminal domain of TEAD4 in complex with the TEAD-interacting $\mathrm{N}$-terminal domain of YAP. The structure reveals that the N-terminal region of YAP is folded into two short helices with an extended loop containing the PXXФP motif in between, while the C-terminal domain of TEAD4 has an immunoglobulin-like fold. YAP interacts with TEAD4 mainly through the two short helices. Point mutations of TEAD4 indicate that the residues important for YAP interaction are required for its transforming activity. Mutagenesis reveals that the PХХФP motif of YAP, although making few contacts with TEAD4, is important for TEAD4 interaction as well as for the transforming activity.

[Keywords: Hippo signaling pathway; TEAD; YAP; organ size control]

Supplemental material is available at http://www.genesdev.org.

Received September 18, 2009; revised version accepted December 11, 2009.

Growth control, which controls the size of the organ/ organism, is a fundamental aspect of multicellular life. Cell growth, proliferation, and apoptosis regulate organ size during development and tissue homeostasis (Conlon and Raff 1999|. Drosophila's Hippo signaling pathway has been delineated as a key mechanism that regulates organ size (Harvey and Tapon 2007; Pan 2007; Saucedo and Edgar 2007; Reddy and Irvine 2008; Zeng and Hong 2008; Zhao et al. 2008a). Several core components of this pathway identified by genetic screening include Hippo (Hpo), Salvador (Sav), Warts (Wts), and Mats (Harvey and Tapon 2007; Saucedo and Edgar 2007; Zeng and Hong 2008). Activation of this pathway induces a kinase cascade that consists of a Ste20-like kinase, Hippo (Harvey et al. 2003; Jia et al. 2003; Pantalacci et al. 2003; Udan et al. 2003; Wu et al. 2003), and the downstream NDR family kinase Warts (Justice et al. 1995; Xu et al. 1995). The Hippo kinase acts in a complex with the WW repeat protein Salvador to phosphorylate and activate Warts (Tapon et al. 2002; Wu et al. 2003; Lai et al. 2005; Wei

\footnotetext{
${ }^{5}$ These authors contributed equally to this work.

Corresponding authors.

${ }^{6}$ E-MAIL haiwei@imcb.a-star.edu.sg; FAX 65-6779-1117.

${ }^{7}$ E-MAIL mcbhwj@imcb.a-star.edu.sg; FAX 65-6779-1117.

Article is online at http://www.genesdev.org/cgi/doi/10.1101/gad.1865310.
}

et al. 2007). Warts, facilitated by binding to a Mob1related regulatory protein, Mats, phosphorylates the transcriptional coactivator Yorkie (Yki), a potent activator of cell growth and proliferation (Dong et al. 2007; Zhao et al. 2007). Upstream of the Hippo kinase cascade, Merlin (Mer) and Expanded (Ex), two membrane-associated FERM domain-containing proteins, have been implicated in linking the protocadherin Fat, a candidate membrane receptor to the Hippo signaling pathway, although the underlying mechanisms are not fully known (Bennett and Harvey 2006; Cho et al. 2006; Hamaratoglu et al. 2006; Silva et al. 2006; Willecke et al. 2006; Tyler and Baker 2007).

The Hippo pathway restricts cell growth and proliferation as well as promotes apoptosis by regulating the nuclear localization of Yki. Mechanistically, Yki is phosphorylated and inactivated by the Warts-Mats complex, thereby suppressing its nuclear accumulation through interaction with 14-3-3 proteins in the cytoplasm. Inactivation of Hippo signaling, or overexpression of Yki, leads to overgrowth of Drosophila tissues. Yki promotes organ growth by stimulating cell proliferation and inhibiting apoptosis. Such regulation is achieved by transcriptional activation of the Hippo pathway target genes, including $c y c E$, diap1, and bantam microRNA (Huang et al. 2005; Nolo et al. 2006; Thompson and Cohen 2006). 
All of the known Hippo pathway components are evolutionally conserved, with the fly Hippo, Salvador, Warts, and Mats corresponding to their mammalian counterparts Mst1/2, WW45, LATS1/2, and Mob1, respectively (Zeng and Hong 2008). YAP is the mammalian homolog of Yki and can functionally rescue Yki mutation in Drosophila (Huang et al. 2005). YAP is a potent growth promoter. Overexpression of YAP increases organ size in Drosophila and saturation cell density of NIH-3T3 cells in culture (Zhao et al. 2007). Several lines of evidence indicated that YAP is an oncogenic protein in mammalian cells (Overholtzer et al. 2006; Zender et al. 2006). First, yap has been shown to be in the human chromosome 11q22 amplicon, which is evident in several human cancers (Overholtzer et al. 2006; Zender et al. 2006). Second, YAP expression and nuclear localization were increased in multiple types of human cancers (Zender et al. 2006; Dong et al. 2007; Zhao et al. 2007; Steinhardt et al. 2008). Third, overexpression of YAP in immortalized epithelial MCF10A cells induced epithelial-mesenchymal transition (EMT), which is often associated with cancer stem cell properties and cancer metastasis (Overholtzer et al. 2006; Polyak and Weinberg 2009). Finally, yap was found to cooperate with the Myc oncogene to stimulate tumor growth in nude mice (Zender et al. 2006). Further support for YAP being an oncogenic protein comes from the observation that transgenic mice with liver-specific YAP overexpression showed a dramatic increase in liver size and eventually developed tumors (Camargo et al. 2007; Dong et al. 2007).

Yki and YAP are transcriptional coactivators and stimulate gene expression by promoting the activity of their cognate transcription factors. Several recent studies performed in Drosophila and mammalian cells showed that the TEAD/TEF family transcription factors partner with Yki/YAP as the downstream targets of the Hippo pathway. Interaction with TEADs is important for Yki/YAPdependent gene induction and growth control (Vassilev et al. 2001; Goulev et al. 2008; Wu et al. 2008; Zhang et al. 2008; Zhao et al. 2008b). In Drosophila, the TEAD/TEF factor Scalloped (Sd) has been shown to interact with Yki and is required for Yki to stimulate tissue growth (Goulev et al. 2008; Wu et al. 2008; Zhang et al. 2008). In mammalian cells, YAP also binds to TEADs, which have four highly homologous proteins (TEAD1-4) in humans and mice (Zhao et al. 2008a). The binding of YAP to TEADs is mediated by the $\mathrm{N}$-terminal domain of YAP and the C-terminal domain of TEAD (Vassilev et al. 2001), and this interaction plays a crucial role in mediating the biological function of YAP. First, activation of a large fraction of YAP-inducible genes is aborted by knockdown of TEADs or disruption of YAP-TEAD interaction by introduction of a TEAD-binding-deficient mutation (Ser 94 to alanine) in YAP (Zhao et al. 2008b). Second, TEADs are crucial for YAP-induced overgrowth, EMT, and oncogenic transformation in MCF10A cells (Zhao et al. 2008b). Third, the phenotype of tead1/tead2 doubleknockout mice is similar to that of yap knockout mice, and genetic data show that TEAD1/TEAD2 and YAP interact with each other in vivo (Sawada et al. 2008). More- over, tead1/tead2 double-knockout embryos exhibit decreased proliferation and increased apoptosis (Sawada et al. 2008). Finally, the importance of YAP and TEAD interaction in cell growth has been implicated in human disease. A heterozygous mutation of a highly conserved tyrosine in the YAP-binding domain of TEAD1 is the underlying cause of the human genetic disease Sveinsson's chorioretinal atrophy (Fossdal et al. 2004).

TAZ is homologous to YAP as well as to fly Yki and shares many functional similarities with YAP (Zhao et al. 2008a). For example, both YAP and TAZ function as oncogenic proteins (Overholtzer et al. 2006; Dong et al. 2007; Zhao et al. 2007; Chan et al. 2008, 2009; Lei et al. 2008). TAZ is also a downstream target regulated by the Hippo pathway, and phosphorylation at the consensus HXRXXS site by the Lats1/2-Mob1 complex results in 14-3-3 protein-mediated cytoplasmic sequestration (Lei et al. 2008). Similar to YAP, TAZ also functions as a transcriptional coactivator and interacts with TEAD (Mahoney et al. 2005). Despite these similarities, the observation that yap and taz knockout mice show different phenotypes (Morin-Kensicki et al. 2006; Hossain et al. 2007; Makita et al. 2008) suggests that YAP and TAZ do not compensate each other and may have distinct regulatory events in cellular and/or developmental processes. Amino acid sequence comparison between YAP and TAZ showed that YAP contains an insertion in the $\mathrm{N}$-terminal TEAD-binding region, including the PXXФP motif (X: any residue; $\Phi$ : hydrophobic residue), which is conserved in all of the YAP homologs but is absent in TAZ (Chan et al. 2009). The lack of this insertion in TAZ may make it function differently from YAP to some extent, but the underlying mechanism remains obscure.

To gain insight into how TEAD interacts with and mediates YAP function, we determined the crystal structure of the C-terminal region of mouse TEAD4 (mTEAD4; residues 210-427) in complex with the N-terminal region of mouse YAP (mYAP; residues 3592). Structure combined with mutagenesis showed that YAP interacts with TEAD4 extensively through three contact areas, with the two short helices of YAP playing the major role in complex formation with TEAD4. The residues involved in the interaction interfaces are critical for cell-transforming activity. Deletion mutation revealed that the loop structure, including the PХХФР motif, which is only conserved in YAP but not in TAZ, is required for TEAD4 interaction as well as for the transforming activity. Altogether, our findings provided the first insight into the structural basis for the interaction between YAP and TEAD, and the important role of the unique loop region in YAP.

\section{Results}

\section{Structure determination}

In this study, the full-length proteins of all four isoforms of TEAD (1-4) and YAP from both mice and humans could not be expressed in soluble form in Escherichia coli. Therefore, we focused on expression of the interacting 
domains of TEAD and YAP mapped by Vassilev et al. (2001). A series of truncated TEAD1-4 and YAP of human and mouse origins was coexpressed, purified, and screened for crystallization conditions. However, only the complex containing the YAP-binding domain of mTEAD4 (residues 210-427) and the N-terminal region of mYAP (residues 35-92) yielded diffraction-quality crystals (Fig. 1A). The crystal structure of the TEAD4-YAP complex was determined by the single-wavelength anomalous dispersion (SAD) method at a resolution of $3.0 \AA$. The final model contains four complexes in the asymmetric unit (AU). Since no substantial differences are observed among the four complexes (pairwise RMSD deviations of $\sim 0.6 \mathrm{~A}$ for all of the $\mathrm{C} \alpha$ atoms), and the polypeptide of YAP in the complex containing chains A (TEAD4) and B (YAP) is more ordered than the rest of the complexes (Supplemental Fig. S1), all subsequent analyses referred to the coordinates of chains A and B. The statistics of structure determination and refinement are summarized in Table 1.

\section{Overall structure}

The overall structure of the TEAD4-YAP complex is shown in Figure 1, B and C. The polypeptide chain of TEAD4 is composed of $12 \beta$ strands and four $\alpha$ helices (Fig. 2A). The $\beta$ strands of TEAD4 adopt an immunoglobulin-like $\beta$-sandwich fold flanked by four short $\alpha$ helices. The two $\beta$ sheets pack against each other with one $\beta$ sheet composed of strands $\beta 1, \beta 2, \beta 5, \beta 8$, and $\beta 9$, and the other consisting of $\beta 3, \beta 4, \beta 6, \beta 7, \beta 10, \beta 11$, and $\beta 12$. A search for structural homologs using the Dali server (Holm et al. 2008) indicated that the YAP-interacting domain of mTEAD4 shares an immunoglobulin-like $\beta$-fold structure with PDE $\delta$ (Hanzal-Bayer et al. 2002), the effector of small GTPase Arl2 (PDB code, $1 \mathrm{KSH} ; Z$ score, 9.7), although they have little sequence identity. Superposition of equivalent $\mathrm{C} \alpha$ atoms of TEAD4 and PDE $\delta$ gives an RMSD of $1.6 \AA$, with the best match being the central $\beta$ sandwich and the notable differences located in the $\alpha$-helical regions (Fig. 1D).

In complex with TEAD4, the N-terminal region of YAP folds into two short helices $(\alpha 1$ and $\alpha 2)$ (Fig. 1B), with an extended loop containing the PХХФР motif (X: any residue; $\Phi$ : hydrophobic residue) (Fig. 2B) in between. The $\alpha 1$ helix binds to a shallow groove formed by $\alpha 3, \alpha 4$, and the $\beta 6-\beta 7$ loop of TEAD4, while the PXXФP-containing loop runs across the body of the $\beta$ sandwich. The $\alpha 2$ helix packs against one side of the $\beta$ sandwich (Fig. 1B,C). Helices $\alpha 1$ and $\alpha 2$ in YAP appear to contribute most significantly to the interaction with TEAD4, while the PXXФP-containing loop seems to play a more minor role.

\section{The TEAD4-YAP interface}

YAP interacts with TEDA4 through three major contact areas (Fig. $1 \mathrm{~B}, \mathrm{C}$ ) with a total buried accessible surface area of $\sim 1300 \AA^{2}$. The first area involves the $\mathrm{N}$-terminal helix
A mTEAD4 131 98

\begin{tabular}{|c|c|c|c|c|}
\hline & 13 & 188 & $218 \quad 247$ & 472 \\
\hline mYAP & TEAD binding & ww & $|w w|$ & \\
\hline
\end{tabular}

B

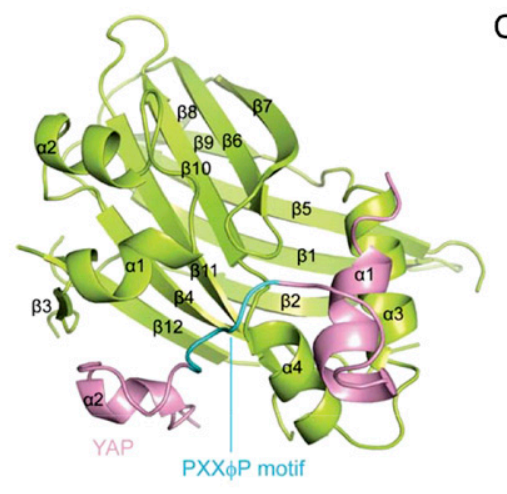

D

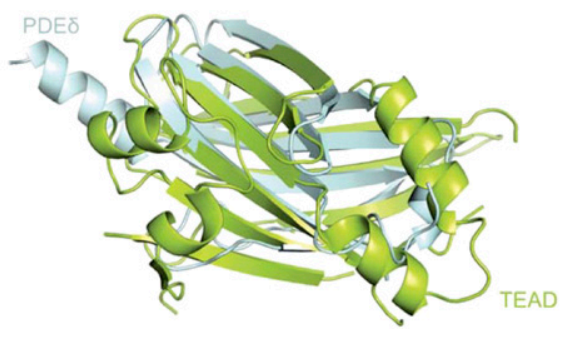

Figure 1. The overall structure of the TEAD4YAP complex. (A) Domain organization of TEAD4 and YAP showing the TEA domain (orange), the YAP-binding domain of mTEAD4 (green), and the TEAD-binding domain (pink) and the WW domains (yellow) of mYAP. (B) A ribbon diagram of the TEAD4-YAP complex. TEAD4 and YAP are shown in green and pink, respectively, and the РХХФР motif in YAP is colored in cyan. Secondary structure for both TEAD4 and YAP are marked. $(C)$ Surface view of TEAD4 (green) with bound YAP in ribbon diagram (pink, with the РХХФР motif in cyan). The view is as in $B$. (D) Superposition of the structures of TEAD4 (green) and PDE (light blue). 
Table 1. Data collection and refinement statistics

\begin{tabular}{|c|c|c|}
\hline Data collection & $\begin{array}{c}\text { Se-Met } \\
\text { TEAD4-YAP }\end{array}$ & $\begin{array}{c}\text { Native } \\
\text { TEAD4-YAP }\end{array}$ \\
\hline $\begin{array}{l}\text { Wavelength }(\AA) \\
\text { Resolution limit }(\AA) \\
\text { Space group }\end{array}$ & $\begin{array}{c}0.9798 \\
20-3.1 \\
\mathrm{P} 2_{1} 2_{1} 2_{1}\end{array}$ & $\begin{array}{l}0.9796 \\
20-3.0 \\
\mathrm{P} 2_{1} 2_{1} 2_{1}\end{array}$ \\
\hline $\begin{array}{l}\text { Unit cell dimensions } \\
\qquad a, b, c(\AA) \\
\alpha, \beta, \gamma\left({ }^{\circ}\right)\end{array}$ & $\begin{array}{l}101.61,148.09 \\
165.57 \\
90,90,90\end{array}$ & $\begin{array}{l}100.98,146.91 \\
165.47 \\
90,90,90\end{array}$ \\
\hline $\begin{array}{l}\text { Unique reflections }(N) \\
\mathrm{I} / \sigma \\
\text { Completeness }(\%) \\
\text { Redundancy } \\
\mathrm{R}_{\text {merge }}\end{array}$ & $\begin{array}{c}50,744 \\
9.3(7.1) \\
99.9(100.0) \\
7.8(6.5) \\
0.09(0.58)\end{array}$ & $\begin{array}{c}58,218 \\
9.5(2.5) \\
99.9(100.0) \\
3.6(3.7) \\
0.06(0.30)\end{array}$ \\
\hline $\begin{array}{l}\text { Refinement } \\
\text { Resolution range }(\AA) \\
\text { Used reflections }(N) \\
\text { Nonhydrogen atoms } \\
\text { (water) } \\
\mathrm{R}_{\text {work }}(\%)^{\mathrm{b}} / \mathrm{R}_{\text {free }}(\%)^{\mathrm{c}}\end{array}$ & & $\begin{array}{c}20-3.0 \\
47,233 \\
7933(226) \\
\\
23.3 / 28.8\end{array}$ \\
\hline $\begin{array}{l}\text { RMSD } \\
\text { Bond lengths }(\AA) \\
\text { Bond angles }\left({ }^{\circ}\right)\end{array}$ & & $\begin{array}{l}0.01 \\
1.50\end{array}$ \\
\hline $\begin{array}{l}\text { Ramachandran plot } \\
\text { Most favored region } \\
\text { Allowed region } \\
\text { Generously allowed region } \\
\text { Disallowed region }\end{array}$ & & $\begin{array}{r}79.8 \% \\
18.1 \% \\
1.9 \% \\
0.2 \%\end{array}$ \\
\hline
\end{tabular}

Values in parentheses indicate the specific values in the highestresolution shell.

${ }^{\mathrm{a}} \mathrm{R}_{\text {merge }}=\Sigma\left|\mathrm{I}_{\mathrm{j}}-\langle\mathrm{I}\rangle\right| / \Sigma \mathrm{I}_{\mathrm{j}}$, where $\mathrm{I}_{\mathrm{j}}$ is the intensity of an individual reflection, and $\langle\mathrm{I}\rangle$ is the average intensity of that reflection.

${ }^{\mathrm{b}} \mathrm{R}_{\text {work }}=\Sigma|| \mathrm{F}_{\mathrm{o}}|-| \mathrm{F}_{\mathrm{c}}|| / \Sigma\left|\mathrm{F}_{\mathrm{c}}\right|$, where $\mathrm{F}_{\mathrm{o}}$ denotes the observed structure factor amplitude, and $F_{c}$ denotes the structure factor amplitude calculated from the model.

${ }^{\mathrm{c}} \mathrm{R}_{\text {free }}$ is as for $\mathrm{R}_{\text {work }}$ but calculated with $5.0 \%$ of randomly chosen reflections omitted from the refinement.

$\alpha 1$ of YAP and helices $\alpha 3$ and $\alpha 4$ of TEAD4 (Fig. 3A). Residues L50, L53, F54, V57, and M58 in $\alpha 1$ of YAP form a hydrophobic patch to interact with the hydrophobic groove formed by residues Y362, F366, the methylene group of K369, L370, L373, M378, V382, and F386 in TEAD4. All of the residues involved in this contact area and the other two interaction sites are highly conserved in YAP and TEADs (Fig. 2).

The second interaction site is mediated mainly by the PXXФP motif of YAP (residues $65-70$ ) and the $\beta 6-\beta 7$ loop and the $\alpha 4-\beta 10$ loop in TEAD4 (Fig. 3B). Residues V65 and P66 of YAP contact F330 of TEAD4 via hydrophobic interactions, while T68 and P70 in YAP make hydrogen bond and van der Waals contacts with N385 and E384 of TEAD4, respectively.

The third contact area is composed of the downstream loop of the РХХФP motif, helix $\alpha 2$ and the following C-terminal region of YAP (residues 71-84), and strands $\beta 4, \beta 11$, and $\beta 12$ of TEAD4 (Fig. 3C). In this interaction site, residues M71, L76, F80, F81, P83, and P84 in YAP form a hydrophobic surface to interact with a hydrophobic groove in TEAD4, which is formed by residues L288, the aliphatic group of K290, W292, V407, the methylene group of Q418, H420, and Y422. In addition to these hydrophobic interactions, van der Waals contacts exist to enhance the interactions on this site. Specifically, M71 in YAP makes van der Waals contacts with K266 and E384 of TEAD4, while R74 in YAP contacts D265 and K266 of TEAD4, and P84 in YAP contacts H420, W292, and Q418 in TEAD4 via van der Waals interactions. Additional van der Waals interactions are made between F81 of YAP and K290 and E409 of TEAD4. Hydrogen bonds are also critical for the interaction of YAP and TEAD4 on the third interaction site. Notably, the side chain of S79 in YAP forms strong hydrogen bonds with the hydroxyl group of Y422 and the side chain of E256, respectively, while R72 is hydrogen-bonded to E409.

Consistent with the residues in the YAP-TEAD4 interface predicted to be important for the interaction between YAP and TEAD4, some mutations in both YAP and TEAD that affected their interaction have been mapped to this interface. For example, mutation of S94 in human YAP (hYAP) and S97 in fly Yki (equivalent to S79 in mYAP) (Supplemental Table S1) to Ala abolished its binding to TEADs and $\mathrm{Sd}$, respectively (Zhao et al. 2008b). S79 is an invariant residue in all of the YAP homologs (Fig. 2B). Substitution of S79 in YAP by any other amino acid would disrupt its hydrogen-bonding network (Fig. 3C), therefore abolishing its interaction with TEADs. Similarly, mutation of P88 to Leu in Yki has been shown to abolish its binding to $\mathrm{Sd}(\mathrm{Wu}$ et al. 2008). Since the equivalent residue in mYAP (P70) only contacts residue E384 in TEAD4 via van der Waals interaction (Fig. 3B), mutation of this residue probably partially disrupts the local peptide conformation of the РХХФР motif, suggesting that keeping the conformation of this motif intact might be important for the interaction of YAP and TEAD4. Sveinsson's chorioretinal atrophy (Fossdal et al. 2004) is a human genetic disease caused by a missense mutation of a highly conserved Y421 (Y422 in mYAP) (Table 2) to His in the YAP-binding domain of human TEAD1. Moreover, mutation of this residue in TEAD1 abolished its interaction with YAP and TAZ (Kitagawa 2007). Our structure showed that, in addition to the hydrogen bond formed between Y422 and S79 mentioned above, Y422 also contacts F80 via a hydrophobic interaction (Fig. 3C). Replacement of this residue by His or any other residue would disrupt its interaction with YAP and account for the observed biochemical and pathological phenotypes.

TAZ is a YAP paralog and shares many functional similarities with YAP. Like YAP, the TEAD-binding domain of TAZ is also located at its $\mathrm{N}$-terminal region. It has been shown that mutations of L31F32, W43R44, L48P49, and F52F53 of TAZ-corresponding to L53F54, M71R72, L76P77, and F80F81 of YAP (Supplemental Table S1), respectively-reduced the binding of TAZ to TEADs (Chan et al. 2009). Our structure showed that all of these residues are involved in interactions with TEAD4 to some extent (Fig. 3). Double mutations of these pairs of residues would be expected to affect the interaction of TAZ with 
Chen et al.

A
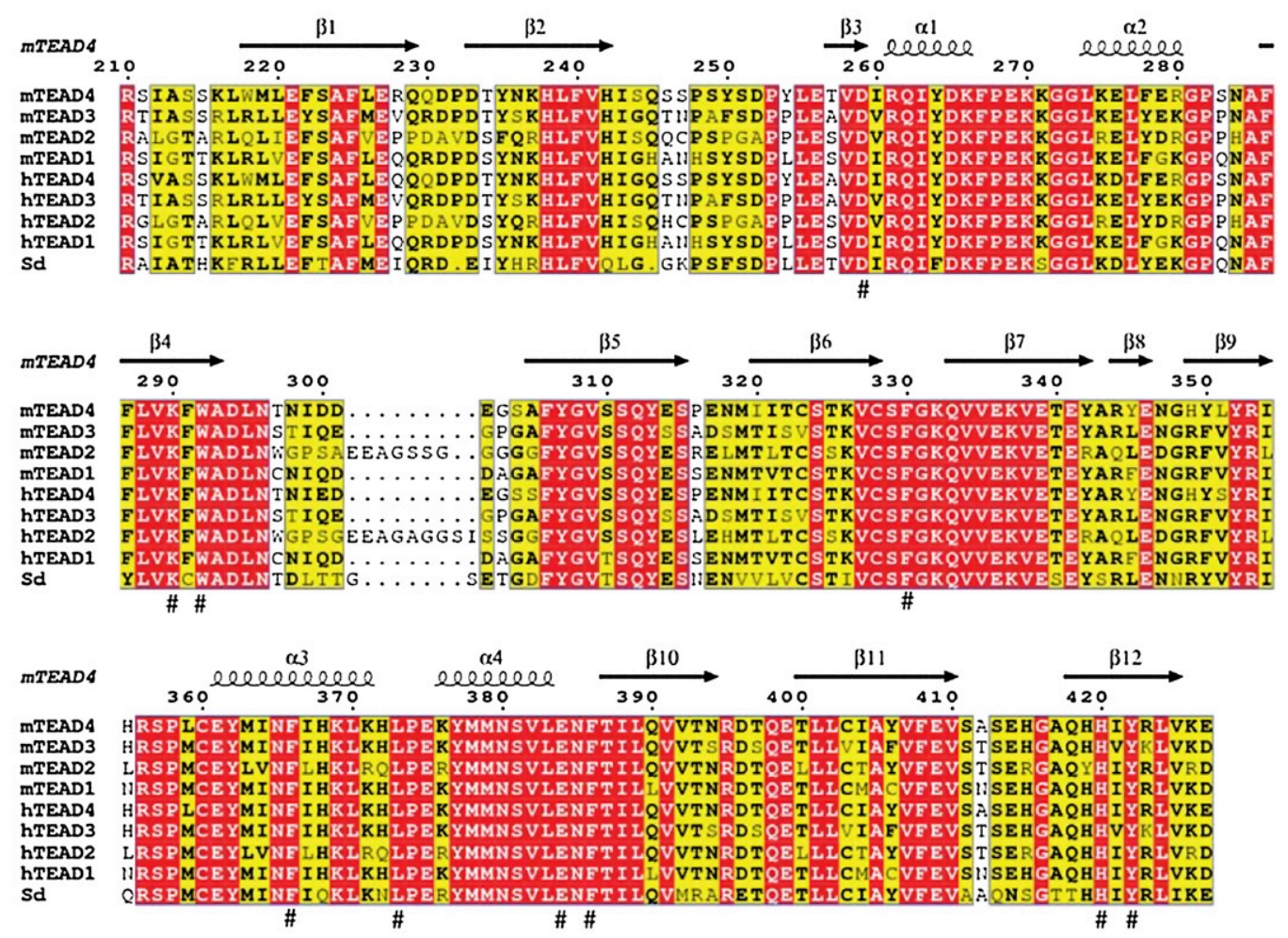

B

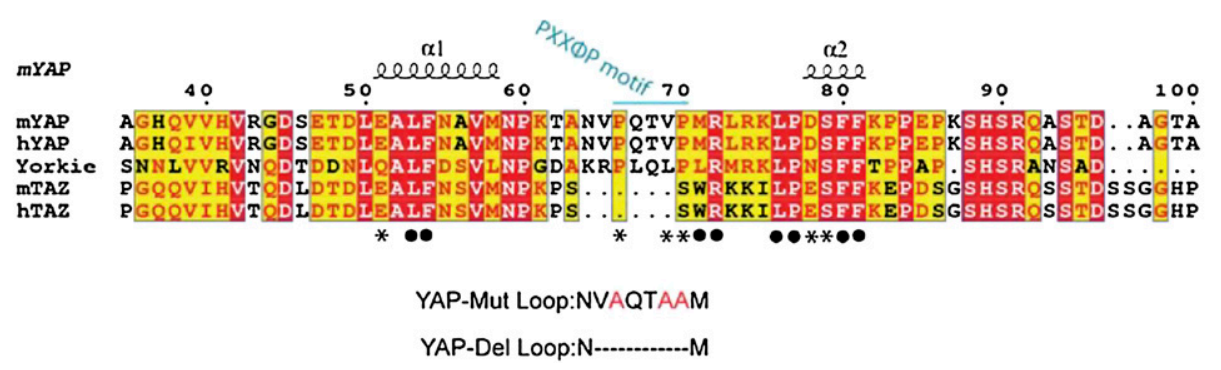

Figure 2. Sequence alignment of TEAD and YAP. (A) Alignment of amino acid sequences of the YAP-binding domains of TEAD1-4 and Sd. $(B)$ Alignment of amino acid sequences of the TEAD-binding domains of YAP/Yorki/TAZ. Secondary structures for mTEAD4 and mYAP are indicated at the top of each alignment. Residues mutated in hTEAD4 and hYAP involved in the TEAD4-YAP interface are marked with the number sign (\#) and an asterisk $\left({ }^{\star}\right)$, respectively, while those mutated in hTAZ are marked with a dot $(\bullet)$ The PXXФP motif unique to YAP/Yorkie is marked with a cyan solid line.

TEADs, providing a structural basis for the previous biochemical and functional observations (Chan et al. 2009).

Identification of residues in the C-terminal region of TEAD4 that are important for interaction with YAP

To further identify the residues in TEAD4 that are important for YAP binding, 10 mutations, based on the structure of the TEAD4-YAP complex, covering the YAPbinding domain of human TEAD4 (hTEAD4) (Fig. 2A), have been made. Each of these mutants (full-length) has a single residue replaced by alanine. These HA-tagged mutants, together with wild-type TEAD4 (TEAD4-WT), were each coexpressed transiently with Flag-tagged YAP in 293 cells. Whole-cell extracts were immunoprecipitated with anti-Flag antibody, and the amounts of coimmunoprecipitated HA-TEAD4 and its mutants were assessed by immunoblotting analysis with anti-HA antibody. As shown in Figure 4A (top panel), mutants D266A, F373A, L380A, E391A, F393A, and H427A (lanes 3,6-10), like TEAD4-WT (lane 2), coimmunoprecipitated well with Flag-YAP. These mutants are thus not obviously affected in their ability to interact with YAP. However, the amount of mutants W299A, Y429A, and K297A (Fig. $4 \mathrm{~A}$, top panel, lanes $4,11,12$ ) coimmunoprecipitated with YAP was dramatically reduced, and hence these mutants 
A
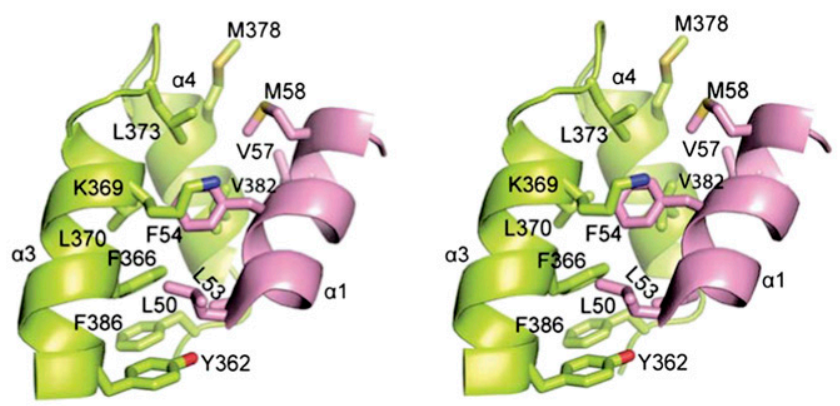

B
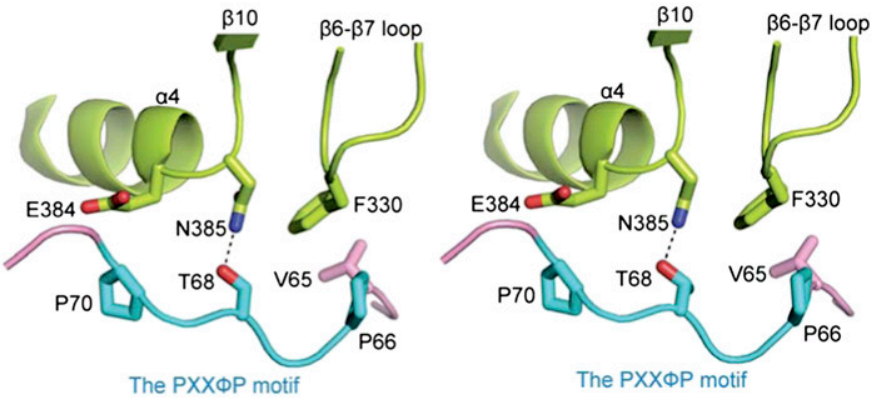

C

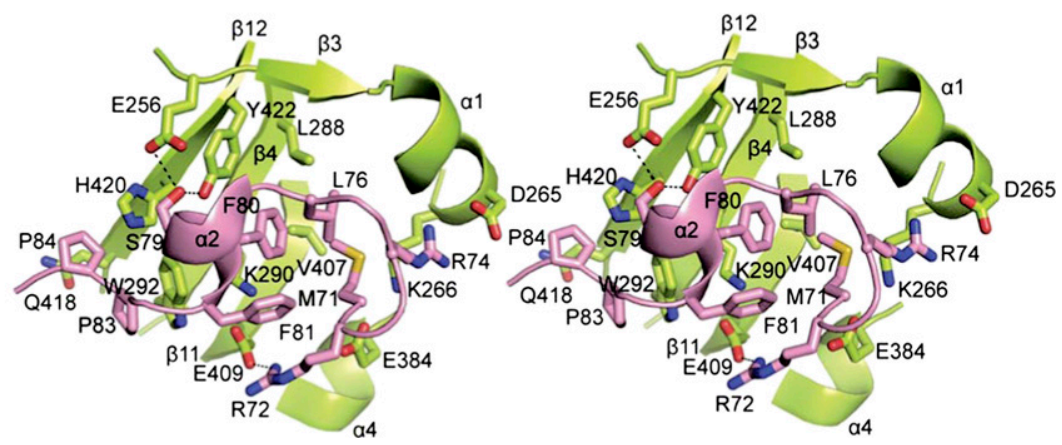

Figure 3. The TEAD4-YAP interface. (A) The first interaction site involving helices $\alpha 3$ and $\alpha 4$ of TEAD 4 and $\alpha 1$ of YAP. $(B)$ The second interaction site involving the РХХФР motif of YAP and the $\alpha 4-\beta 10$ loop and the $\beta 6-\beta 7$ loop of TEAD4. (C) The third interaction site containing residues $71-85$ of YAP and $\beta 4, \beta 10, \beta 11, \beta 12$ of TEAD4. TEAD4 and YAP are shown in green and pink, respectively. Residues in TEAD4 and those in YAP involved in the interface are shown in stick models. Hydrogen bonds are shown in black broken lines. have significantly lost their ability to interact with YAP. Mutant F337A (Fig. 4A, top panel, lane 5) has some reduced ability to interact with YAP. These mutagenesis results show that these residues are indeed important for the YAP/TEAD4 interaction in the context of the fulllength proteins in vivo, consistent with our structural analysis (Fig. 3; Table 2).

\section{TEAD4 mutants defective in YAP interaction lose transforming ability}

Our previous study indicates that TEAD4 has a weak but detectable transforming activity (Chan et al. 2009). To examine whether interaction with YAP is important for TEAD4's transforming ability, the oncogenic property of different TEAD4 mutants was examined, along with wild-type TEAD4, by their abilities to confer anchorageindependent growth of MCF10A in soft agar. D266A, F373A, L380A, E391A, F393A, and H427A, whose interactions with YAP were not significantly affected by the mutations (Fig. 4A, top panel), exhibited transforming abilities that are $\sim 60 \%$ of TEAD4-WT (Fig. $4 \mathrm{~B}, \mathrm{C}$ ). How- ever K297A, W299A, and Y429A, whose interactions with YAP were greatly abrogated by the mutations (Fig. 4A), essentially lost the transformation ability (Fig. 4B,C). F337A, which has partially lost the interaction with YAP, has reduced ability to transform MCF10A cells (Fig. 4).

Table 2. Residues mutated in hTEAD4 and their counterparts in mTEAD4

\begin{tabular}{lcc}
\hline $\begin{array}{l}\text { Residues mutated } \\
\text { in hTEAD4 }\end{array}$ & $\begin{array}{c}\text { Mutation of hTEAD1 in } \\
\text { Sveinsson's chorioretinal } \\
\text { atrophy disease }\end{array}$ & $\begin{array}{c}\text { Equivalent } \\
\text { residues in } \\
\text { mTEAD4 }\end{array}$ \\
\hline D266 & & D259 \\
K297 & & K290 \\
W299 & & W292 \\
F337 & F330 \\
F373 & & F366 \\
L380 & & L373 \\
E391 & & E384 \\
F393 & & F386 \\
H427 & & H420 \\
Y429 & & Y422 \\
\hline
\end{tabular}




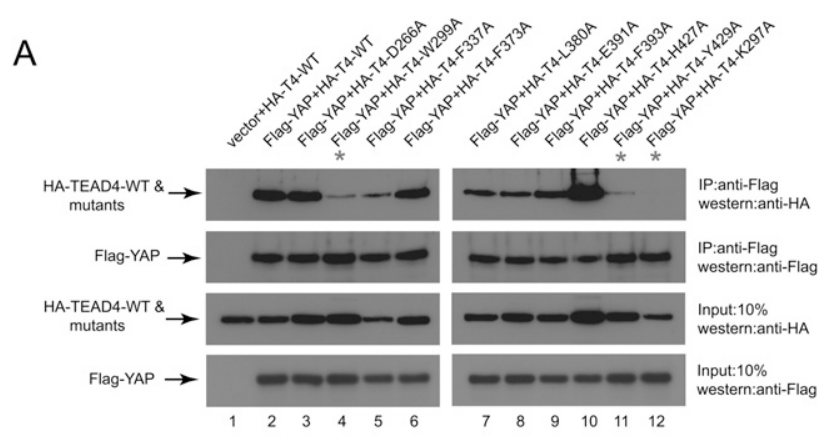

B

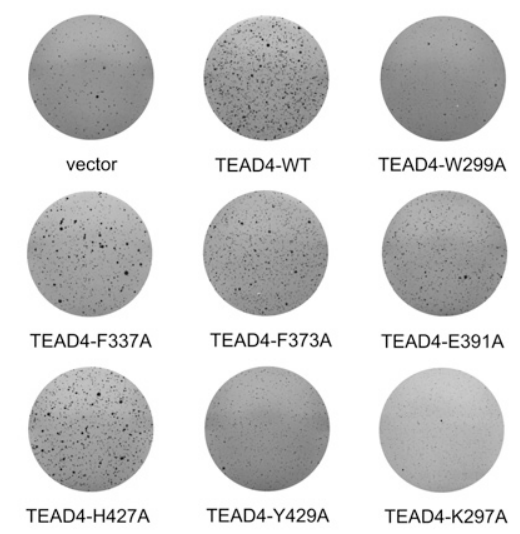

C

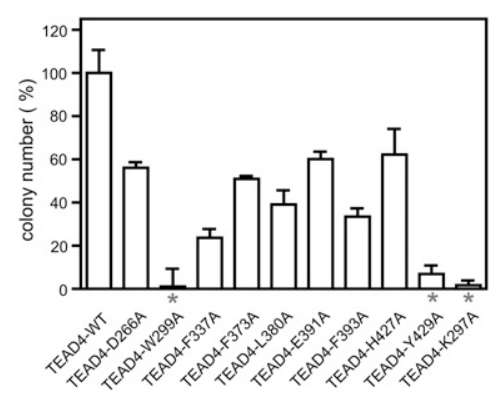

Figure 4. Residues in TEAD4 involved in interactions with YAP are important for anchorage-independent cell growth. $(A)$ Whole-cell lysates from 293 cells transiently expressing FlagYAP, HA-TEAD4-WT, and different HA-TEAD4 mutants were immunoprecipitated with anti-Flag, and the immunoprecipitates were probed with anti-HA to detect coimmunoprecipitated HA-TEAD4 and its mutants. The blots were stripped and reprobed with anti-Flag-HRP to detect immunoprecipitated Flag-YAP. Mutations of residues W299 (lane 4), Y429 (lane 11), and K297 (lane 12) of hTEAD4 disrupted interaction with hYAP. These three mutants are highlighted with asterisks. (B) Transformation ability assay for mutants of hTEAD4. Soft agar assays were done using MCF10A cells transduced with expressing vector (vector) and vectors for expressing TEAD4-WT and the indicated TEAD4 mutants. The colonies grown up in soft agar after 1 mo were stained with thiazolyl blue tetrazolium bromide. $(C)$ Quantification of the colony number from three independent soft agar assays. The colony numbers were obtained after subtracting those of vector control and presented as percentage relative to TEAD4-WT, which was arbitrarily set as $100 \%$.
The correlation of the ability to interact with YAP and the transforming potential of TEAD4 suggests that interaction with YAP is essential for TEAD4-mediated transformation.

The PХХФP-containing loop of YAP is essential for TEAD4 interaction as well as for the transforming activity

Since the РХХФР-containing loop is shown to play a minor role in interaction with TEAD4 (Fig. 3B), yet mutation of P88 in Yki (which corresponds to P70 in the РХХФР motif of mYAP) disrupted its binding to $\mathrm{Sd}(\mathrm{Wu}$ et al. 2008), we decided to further investigate the importance of this unique loop in interaction with TEAD4 and in the transforming activity by mutagenesis of human YAP (hYAP). Residue P81 (equivalent to P66 in mYAP) was replaced by alanine, and the loop VPQTVP (Fig. 2B) was either completely deleted (YAP-Del-Loop) or mutated to VAQTAA (YAP-Mut-Loop). In addition, E66 and D93 (E51 and D78 in mYAP) located outside of this loop were mutated to Ala as controls. These five mutants and YAP-WT were transiently coexpressed in 293 cells with HA-TEAD4, and the cell extracts were immunoprecipitated with anti-Flag and immunoblotted with anti-HA to detect the amount of coimmunoprecipitated HA-TEAD4. All three single-point mutations (E66A, P81A, and D93A) interact efficiently with TEAD4 (Fig. 5A, top panel, lanes 10-12), consistent with the observations that these residues either are not involved in binding to TEAD4 or make quite few contacts with TEAD4 (Fig. 3).

Unexpectedly, mutation or deletion of the YAP-specific loop strongly affected the interaction of YAP with TEAD4, with the YAP-Del-Loop mutant completely losing interaction with TEAD4 (Fig. 5A, top panel, lanes 13,14). These results strongly suggest that the intact loop structure in YAP is essential for its binding to TEAD4. P85 (P70 in mYAP) in the РХХФР motif may be required to retain a conformation of the loop structure in a manner favoring the efficient binding of YAP to TEAD4.

To examine the functional relevance of the importance of this loop structure of YAP, the transforming abilities of the mutants were examined by stably expressing in MCF10A cells followed by assessing their abilities to drive anchorage-independent growth in soft agar. As shown in Figure 5, B and C, cells expressing YAP-E66A, YAP-P81A, and YAP-D93A showed similar abilities to grow in soft agar as compared with cells expressing YAPWT. However, the YAP-Mut-Loop mutant, which has reduced interaction with TEAD4, displayed significantly reduced ability to grow in soft agar, while the YAP-DelLoop mutant almost completely lost the ability to grow in soft agar. These results suggest that the unique loop of YAP is not only essential for its interaction with TEAD4, but it is also important for YAP to transform MCF10A cells.

\section{Discussion}

The crystal structure of the TEAD4-YAP complex presented here shows that YAP binds to TEAD4 through 


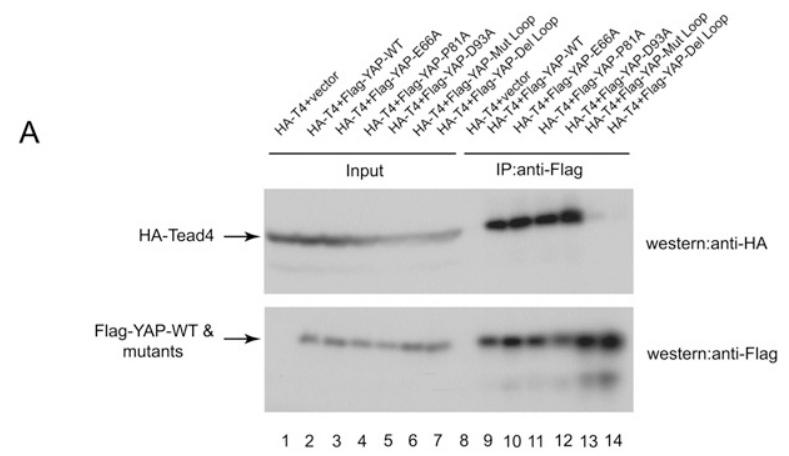

B

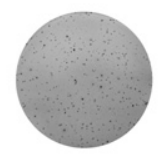

vector

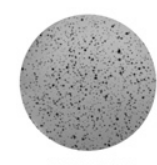

YAP-WT
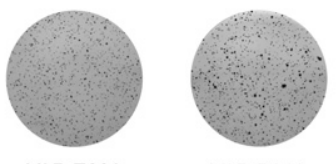

YAP-P81A

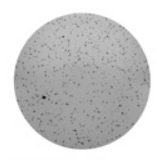

YAP-Del Loop
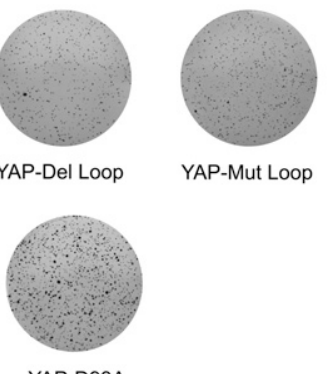

C

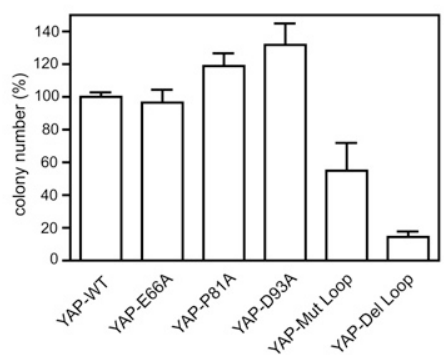

Figure 5. Importance of the РХХФР loop in interaction with TEAD4 and the transforming activity. $(A)$ Whole-cell lysates from 293 cells transiently expressing HA-TEAD4, Flag-YAP, and Flag-YAP mutants were immunoprecipitated with anti-Flag, and the immunoprecipitates were probed with anti-HA to detect coimmunoprecipitated HA-TEAD4. The blots were stripped and reprobed with anti-Flag-HRP to detect immunoprecipitated Flag-YAP and its mutants. Single mutations of residues P81, V84, and P85 to Ala did not disrupt interaction with TEAD4, but mutation or deletion of the PXXФP-containing loop in YAP disrupted interaction with TEAD4. (B) Transforming activities of YAP mutants. Soft agar assays were done as in Figure 4B, using MCF10A cells transduced with expressing vector, vectors for expressing Flag-YAP-WT, and the indicated mutants of YAP. $(C)$ Quantification of the colony number from $A$. The detail is as described in the legend for Figure 4C.

three distinct regions of its $\mathrm{N}$-terminal domain; namely, two short helical segments and an intervening loop containing the РХХФР motif. The two short helices contribute most significantly to the interaction with TEAD4, while the РХХФР-containing loop plays only a limited role in complex formation with TEAD4. The interaction of YAP with TEAD4 is similar to that observed in the structure of YAP2 in complex with TEAD1 (Li et al. 2010).
The $\mathrm{Y} 421 \mathrm{H}$ mutation of human TEAD1 that is the underlying cause of human Sveinsson's chorioretinal atrophy disease (Fossdal et al. 2004) is mapped in the TEAD4-YAP interface (equivalent to Y422 of mTEAD4), suggesting that the disease phenotype is likely due to the defective binding to YAP caused by this mutation. Consistent with this view, mutation of this tyrosine residue in TEADs abolished their interaction with and their activation by YAP (Kitagawa 2007). Further structurebased mutagenesis identified four residues-K297, W299, F337, and Y429 in hTEAD4 (corresponding to K290, W292, F330, and Y422, respectively, in mTEAD4)whose Ala mutations substantially reduced the binding to YAP. More importantly, the binding defects of these mutants are correlated with dramatically reduced transforming activities, suggesting that the transforming ability of TEAD4 is likely mediated by its ability to engage direct interaction with endogenous YAP.

The structure of the TEAD4-YAP complex also provided rational explanations for the previous biochemical observations in YAP. Mutation of S94 to Ala of hYAP (S79 in mYAP) abolished the binding of YAP to TEADs. S79 is hydrogen-bonded to Y422 in TEAD4 (Fig. 3C). Furthermore, several residues mutated in TAZ / which affected its binding to TEADs) (Chan et al. 2009), are conserved in YAP, and are shown to be involved in direct interaction with TEAD4 to different extents. Mutation of S79 and these residues would disrupt or at least reduce the binding of YAP to TEAD4. Interestingly, our mutational data showed that the loop containing the PXXФP motif in YAP, which is a unique feature in YAP but not in TAZ (Fig. 2B), is essential for TEAD4 interaction as well as for the transforming activity. Moreover, mutation of P88 to Leu (P70 in mYAP) in Yki abolished its binding to Sd (Wu et al. 2008), although this residue only makes one van der Waals contact with TEAD4 (Fig. 3B). Altogether, these results suggest that the integrity of the loop structure in YAP is essential for interaction with TEAD. Since this loop in YAP plays only a minor role in mediating the interaction with TEAD4, it is unlikely that the loss of the TEAD4-YAP interaction is due to the disrupted interaction involving this loop. One simple explanation for these observations is that the two conserved proline residues, particularly P70 (mYAP numbering), in this loop may restrict the movement of this loop and lock it in a certain conformation. Through this way, the loop may coordinate/orchestrate the other two main interacting areas provided by the $\alpha 1$ helix and $\alpha 2$ helix, respectively, allowing YAP to bind efficiently to TEAD4. However, this explanation is complicated by the observations that TAZ, which lacks this loop, still binds to TEAD, whereas the YAP-Del-Loop mutant, which essentially recapitulates the wild-type TEAD-binding region of TAZ, failed to bind to TEAD. Given that the two helices involved in interaction with TEAD4 are highly conserved in both YAP and TAZ, the binding of TAZ to TEAD most likely involves two helices in a manner similar to that observed in the TEAD4-YAP complex, but how these two helices are coordinated to achieve efficient binding remains to be defined, presumably through residues outside these two 
helical regions. Further structural and biochemical studies are required to clarify this apparent paradox.

The Hippo pathway controls cell growth, and dysregulation of Hippo signaling has been implicated in mammalian tumorigenesis (Harvey and Tapon 2007; Pan 2007; Saucedo and Edgar 2007; Zeng and Hong 2008). YAP is the downstream target of the Hippo pathway, and the interaction of TEADs and YAP mediates the output of the Hippo pathway, including growth control and cancer development (Goulev et al. 2008; Wu et al. 2008; Zhang et al. 2008; Zhao et al. 2008b). The abnormal activation of YAP has been associated with multiple types of cancer (Overholtzer et al. 2006; Zender et al. 2006; Dong et al. 2007; Zhao et al. 2007). The crystal structure of the TEAD4-YAP complex presented here and the crystal structure of YAP2 in complex with TEAD1 (Li et al. 2010) not only elucidate the structural basis for the TEAD/YAP interaction, but also may provide a new strategy for cancer therapeutics by disrupting the TEAD/YAP interaction. Given that only a small region in the $\mathrm{N}$-terminal region of YAP is involved in the interaction with TEAD4, one way to block the binding of YAP to TEAD4 is to design a peptide molecule that mimics the region of YAP bound to TEAD4 in the structure. Our structure also provides a starting point for further structural, biochemical, and genetic studies to elucidate the molecular mechanism of how YAP and TAZ activate transcription.

\section{Materials and methods}

Cloning, protein expression, and purification

mTEAD4 (210-427) and His-tagged mYAP (35-92) were cloned into the pETDuet-1 vector (Novagen), and were coexpressed in E. coli BL21 (DE3) strain grown at $18^{\circ} \mathrm{C}$ after induction with $0.1 \mathrm{mM}$ IPTG. The TEAD4-YAP complex was purified on TALON $\mathrm{Co}^{2+}$ resin. The complex was further purified by Superdex $75 \mathrm{gel}$ filtration column in a buffer containing $20 \mathrm{mM}$ Tris $(\mathrm{pH} 8.0)$, $150 \mathrm{mM} \mathrm{NaCl}$, and $5 \mathrm{mM} \beta$-mercaptoethanol. The complex was concentrated to $\sim 10 \mathrm{mg} / \mathrm{mL}$ for crystallization. The SeMetlabeled complex was expressed in a minimal medium containing $20 \mathrm{mg} / \mathrm{L}$ SeMet, and was purified in the same way as the native protein.

\section{Crystallization and structure determination}

Crystallization screening was carried out with a Phoenix robot. Both the native and SeMet-substituted TEAD4-YAP complexes were crystallized using the buffer containing $0.1 \mathrm{M} \mathrm{Na}_{3}$ citrate (pH 5.6), 6\% PEG10K, and 0.1 M Mg acetate. The crystals were transferred to a cryo-buffer $\left(0.1 \mathrm{M} \mathrm{Na}_{3}\right.$ citrate at $\mathrm{pH} 5.6,25 \%$ glycerol, 6\% PEG10K, and $0.1 \mathrm{M} \mathrm{Mg}$ acetate) and flash-frozen in liquid nitrogen. Native and SeMet SAD data were collected at Australian and European Synchrotron Radiation Facilities, respectively. Crystals belong to space group $\mathrm{P} 2{ }_{1} 2_{1} 2_{1}$ with cell parameters $a=101.0 \AA, b=147.0 \AA, c=165.5 \AA$, and contain four complexes per AU. The data were processed with the CCP4 package (Collaborative Computational Project 1994).

Twenty out of the expected 32 Se sites were located by using the program SnB (Miller et al. 1994). Refinement of the heavyatom sites and phasing were carried out using SHARP (De la Fortelle and Bricogne 1997). After density modification and sol- vent flattening, a partial model built from ARP/wARP (Perrakis et al. 1999) was used for manual model building with the program Coot (Emsley and Cowtan 2004). Crystallographic refinement was performed with the programs CNS (Brunger et al. 1998) and REFMAC5 (Murshudov et al. 1997) using the native data set at a resolution of $3.0 \AA$. The final model has a good stereochemistry, with a free $\mathrm{R}$ factor of $28.8 \%$ and an $\mathrm{R}$ factor of $23.3 \%$. Several regions of the polypeptide chains are not visible in the electron density map and are assumed to be disordered; namely, residue chain A: 244-252, 299-301, and 414-416; chain C: 246-251; chain E: 414-416; and chain G: 246-250, 270-271, 299-306, 329-337, and 412-416 in TEAD4, and residue chain B: 35-46 and 87-92; chain D: 35-47 and 83-92; chain F: 35-47 and 84-92; and chain H: 35-64 and 84-92. Statistics for data collection and refinement are summarized in Table 1.

\section{Cell lines}

MCF10A cells were purchased from American Type Culture Collection and were cultured in Dulbecco's modified Eagle's medium supplemented with $5 \%$ horse serum, $20 \mathrm{ng} / \mathrm{mL}$ epidermal growth factor, $0.5 \mu \mathrm{g} / \mathrm{mL}$ hydrocortisone, $100 \mathrm{ng} / \mathrm{mL}$ cholera toxin, and $10 \mu \mathrm{g} / \mathrm{mL}$ insulin and penicillin/streptomycin.

\section{Plasmids for transfection studies}

The cDNAs of human YAP were from MCG clones 46148. Flagtagged YAP was constructed by PCR using the MCG clones and was cloned into pBABEpuro retroviral vector. HA-TEAD4 was constructed by PCR using the Image clone 3913870 and was cloned into retroviral vector pBABEpuro. Single or triple amino acids or deletion mutations were introduced into human YAP and human TEAD4 coding regions by PCR.

\section{Antibodies}

EZview Red anti-Flag M2 affinity gel and anti-Flag M2-peroxidase (anti-Flag-HRP) mouse antibodies were from Sigma. The anti-HA-peroxidase (anti-HA-HRP) antibody was from Roche.

\section{Retrovirus generation and infection}

The amphotropic Phoenix packaging cells (Nolan's Laboratory at Stanford University) were transfected with the indicated retroviral vectors using Fugene 6 according to the manufacturer's instructions (Roche). After $48 \mathrm{~h}$, the retroviral supernatants were collected, filtered $(0.45 \mu \mathrm{m}$; Millipore), and added onto the target cells in the presence of $5 \mu \mathrm{g} / \mathrm{mL}$ polybrene (Sigma-Aldrich) for 6-8 h. Infection was done twice. After infection, the cells were selected with puromycin $(1 \mu \mathrm{g} / \mathrm{mL})$ for a week before being analyzed for protein expression by immunoblotting and oncogenesis by soft agar assays.

\section{Anchorage-independent growth in soft agar}

We plated $1.5 \mathrm{~mL}$ of $0.5 \%$ agar (electrograde ultrapure; Invitrogen) supplemented with culture medium for MCF10A cells in six-well plates as the bottom agar. Fifty-thousand cells were mixed with $1.5 \mathrm{~mL}$ of $0.35 \%$ agar-supplemented MCF10A medium and plated onto the solidified bottom agar. One milliliter of medium was added on top of the solidified agar layers, and the colonies were allowed to grow in the incubator for $1 \mathrm{mo}$ at $37^{\circ} \mathrm{C}, 5 \% \mathrm{CO}_{2}$. The colonies were stained with thiazolyl blue tetrazolium bromide, and images were scanned. Colonies were quantified using the ImageJ program. 


\section{Immunoprecipitation}

Human embryonic kidney 293 cells transiently transfected with the indicated plasmids were washed once with ice-cold PBS and were subsequently lysed in ice-cold lysis buffer ( $150 \mathrm{mM} \mathrm{NaCl}$, $50 \mathrm{mM}$ Tris- $\mathrm{HCl}$ at $\mathrm{pH} 7.4,0.5 \%$ NP-40, protease inhibitor cocktail [Roche]). After clearance by a spin $(10,000 \mathrm{~g}, 15 \mathrm{~min})$, a fixed amount of whole-cell lysates was incubated with Ezview Red anti-Flag M2 affinity gel overnight and washed twice with lysis buffer with $500 \mathrm{mM} \mathrm{NaCl}$, followed by three times with lysis buffer. The precipitated proteins were resolved on SDSpolyacrylamide gels and blotted onto nitrocellulose membrane. The filters were blocked in 5\% skim milk in PBS and were probed with anti-HA-HRP antibody. Signals were visualized using Supersignal (Pierce). The blots were stripped and reprobed with anti-Flag-HRP to detect immunoprecipitated Flag-YAP or Flag-TAZ.

\section{Accession numbers}

The coordinates and structure factor amplitudes of the TEAD4YAP complex have been deposited in the Protein Data Bank with accession number 3JUA.

\section{Acknowledgments}

We thank the beamline scientists at Australian and European Synchrotron Radiation Facilities for their assistance during data collection. This work is financially supported by the Agency for Science, Technology, and Research $\left(\mathrm{A}^{\star} \mathrm{Star}\right)$ in Singapore.

\section{References}

Bennett FC, Harvey KF. 2006. Fat cadherin modulates organ size in Drosophila via the Salvador/Warts/Hippo signaling pathway. Curr Biol 16: 2101-2110.

Brunger AT, Adams PD, Clore GM, DeLano WL, Gros P, GrosseKunstleve RW, Jiang JS, Kuszewski J, Nilges M, Pannu NS, et al. 1998. Crystallography \& NMR system: A new software suite for macromolecular structure determination. Acta Crystallogr D Biol Crystallogr 54: 905-921.

Camargo FD, Gokhale S, Johnnidis JB, Fu D, Bell GW, Jaenisch R, Brummelkamp TR. 2007. YAP1 increases organ size and expands undifferentiated progenitor cells. Curr Biol 17: 2054-2060.

Chan SW, Lim CJ, Guo K, Ng CP, Lee I, Hunziker W, Zeng Q, Hong W. 2008. A role for TAZ in migration, invasion, and tumorigenesis of breast cancer cells. Cancer Res 68: 25922598.

Chan SW, Lim CJ, Loo LS, Chong YF, Huang C, Hong W. 2009. TEADs mediate nuclear retention of TAZ to promote oncogenic transformation. J Biol Chem 284: 14347-14358.

Cho E, Feng Y, Rauskolb C, Maitra S, Fehon R, Irvine KD. 2006. Delineation of a fat tumor suppressor pathway. Nat Genet 38: $1142-1150$.

Collaborative Computational Project. 1994. The CCP4 suite: Programs for protein crystallography. Acta Crystallogr D Biol Crystallogr 50: 760-763.

Conlon I, Raff M. 1999. Size control in animal development. Cell 96: 235-244.

De la Fortelle E, Bricogne G. 1997. Maximum-likelihood heavyatom parameter refinement for Multiple Isomorphous Replacement and Multiwavelength Anomalous Diffraction method. Methods Enzymol 276: 472-494.

Dong J, Feldmann G, Huang J, Wu S, Zhang N, Comerford SA, Gayyed MF, Anders RA, Maitra A, Pan D. 2007. Elucidation of a universal size-control mechanism in Drosophila and mammals. Cell 130: 1120-1133.

Emsley P, Cowtan K. 2004. Coot: Model-building tools for molecular graphics. Acta Crystallogr D Biol Crystallogr 60: 2126-2132.

Fossdal R, Jonasson F, Kristjansdottir GT, Kong A, Stefansson H, Gosh S, Gulcher JR, Stefansson K. 2004. A novel TEAD1 mutation is the causative allele in Sveinsson's chorioretinal atrophy (helicoid peripapillary chorioretinal degeneration). Hum Mol Genet 13: 975-981.

Goulev Y, Fauny JD, Gonzalez-Marti B, Flagiello D, Silber J, Zider A. 2008. SCALLOPED interacts with YORKIE, the nuclear effector of the hippo tumor-suppressor pathway in Drosophila. Curr Biol 18: 435-441.

Hamaratoglu F, Willecke M, Kango-Singh M, Nolo R, Hyun E, Tao C, Jafar-Nejad H, Halder G. 2006. The tumour-suppressor genes NF2/Merlin and Expanded act through Hippo signalling to regulate cell proliferation and apoptosis. Nat Cell Biol 8: 27-36.

Hanzal-Bayer M, Renault L, Roversi P, Wittinghofer A, Hillig RC. 2002. The complex of Arl2-GTP and PDE $\delta$ : From structure to function. $E M B O J$ 21: 2095-2106.

Harvey K, Tapon N. 2007. The Salvador-Warts-Hippo pathway-An emerging tumour-suppressor network. Nat Rev Cancer 7: 182-191.

Harvey KF, Pfleger CM, Hariharan IK. 2003. The Drosophila Mst ortholog, hippo, restricts growth and cell proliferation and promotes apoptosis. Cell 114: 457-467.

Holm L, Kaariainen S, Rosenstrom P, Schenkel A. 2008. Searching protein structure databases with DaliLite v.3. Bioinformatics 24: 2780-2781.

Hossain Z, Ali SM, Ko HL, Xu J, Ng CP, Guo K, Qi Z, Ponniah S, Hong W, Hunziker W. 2007. Glomerulocystic kidney disease in mice with a targeted inactivation of Wwtrl. Proc Natl Acad Sci 104: 1631-1636.

Huang J, Wu S, Barrera J, Matthews K, Pan D. 2005. The Hippo signaling pathway coordinately regulates cell proliferation and apoptosis by inactivating Yorkie, the Drosophila homolog of YAP. Cell 122: 421-434.

Jia J, Zhang W, Wang B, Trinko R, Jiang J. 2003. The Drosophila Ste20 family kinase dMST functions as a tumor suppressor by restricting cell proliferation and promoting apoptosis. Genes \& Dev 17: 2514-2519.

Justice RW, Zilian O, Woods DF, Noll M, Bryant PJ. 1995. The Drosophila tumor suppressor gene warts encodes a homolog of human myotonic dystrophy kinase and is required for the control of cell shape and proliferation. Genes \& Dev 9: 534 546.

Kitagawa M. 2007. A Sveinsson's chorioretinal atrophy-associated missense mutation in mouse Teadl affects its interaction with the co-factors YAP and TAZ. Biochem Biophys Res Commun 361: 1022-1026.

Lai ZC, Wei X, Shimizu T, Ramos E, Rohrbaugh M, Nikolaidis N, Ho LL, Li Y. 2005. Control of cell proliferation and apoptosis by mob as tumor suppressor, mats. Cell 120: 675-685.

Lei QY, Zhang H, Zhao B, Zha ZY, Bai F, Pei XH, Zhao S, Xiong Y, Guan KL. 2008. TAZ promotes cell proliferation and epithelial-mesenchymal transition and is inhibited by the Hippo pathway. Mol Cell Biol 28: 2426-2436.

Li Z, Zhao B, Wang P, Chen F, Dong Z, Yang H, Guan K-L, Xu Y. 2010. Structural insights into the YAP and TEAD complex. Genes \& Dev (this issue). doi: 10.1101/gad.1865810.

Mahoney WM Jr, Hong JH, Yaffe MB, Farrance IK. 2005. The transcriptional co-activator TAZ interacts differentially with transcriptional enhancer factor-1 (TEF-1) family members. Biochem J 388: 217-225. 
Makita R, Uchijima Y, Nishiyama K, Amano T, Chen Q, Takeuchi T, Mitani A, Nagase T, Yatomi Y, Aburatani H, et al. 2008. Multiple renal cysts, urinary concentration defects, and pulmonary emphysematous changes in mice lacking TAZ. Am I Physiol Renal Physiol 294: F542-F553. doi: 10.1152/ajprenal.00201.2007.

Miller R, Gallo SM, Khalak HG, Weeks CM. 1994. SnB: Crystal structure determination via shake-and-bake. J Appl Crystallogr 27: 613-621.

Morin-Kensicki EM, Boone BN, Howell M, Stonebraker JR, Teed J, Alb JG, Magnuson TR, O’Neal W, Milgram SL. 2006. Defects in yolk sac vasculogenesis, chorioallantoic fusion, and embryonic axis elongation in mice with targeted disruption of Yap65. Mol Cell Biol 26: 77-87.

Murshudov GN, Vagin AA, Dodson EJ. 1997. Refinement of macromolecular structures by the maximum-likelihood method. Acta Crystallogr D Biol Crystallogr 53: 240-255.

Nolo R, Morrison CM, Tao C, Zhang X, Halder G. 2006. The bantam microRNA is a target of the hippo tumor-suppressor pathway. Curr Biol 16: 1895-1904.

Overholtzer M, Zhang J, Smolen GA, Muir B, Li W, Sgroi DC, Deng CX, Brugge JS, Haber DA. 2006. Transforming properties of YAP, a candidate oncogene on the chromosome 11q22 amplicon. Proc Natl Acad Sci 103: 1240512410 .

Pan D. 2007. Hippo signaling in organ size control. Genes \& Dev 21: 886-897.

Pantalacci S, Tapon N, Leopold P. 2003. The Salvador partner Hippo promotes apoptosis and cell-cycle exit in Drosophila. Nat Cell Biol 5: 921-927.

Perrakis A, Morris R, Lamzin VS. 1999. Automated protein model building combined with iterative structure refinement. Nat Struct Biol 6: 458-463.

Polyak K, Weinberg RA. 2009. Transitions between epithelial and mesenchymal states: Acquisition of malignant and stem cell traits. Nat Rev Cancer 9: 265-273.

Reddy BV, Irvine KD. 2008. The Fat and Warts signaling pathways: New insights into their regulation, mechanism and conservation. Development 135: 2827-2838.

Saucedo LJ, Edgar BA. 2007. Filling out the Hippo pathway. Nat Rev Mol Cell Biol 8: 613-621.

Sawada A, Kiyonari H, Ukita K, Nishioka N, Imuta Y, Sasaki H. 2008. Redundant roles of Tead1 and Tead2 in notochord development and the regulation of cell proliferation and survival. Mol Cell Biol 28: 3177-3189.

Silva E, Tsatskis Y, Gardano L, Tapon N, McNeill H. 2006. The tumor-suppressor gene fat controls tissue growth upstream of expanded in the hippo signaling pathway. Curr Biol 16: 2081-2089.

Steinhardt AA, Gayyed MF, Klein AP, Dong J, Maitra A, Pan D, Montgomery EA, Anders RA. 2008. Expression of Yes-associated protein in common solid tumors. Hum Pathol 39: 1582-1589.

Tapon N, Harvey KF, Bell DW, Wahrer DC, Schiripo TA, Haber DA, Hariharan IK. 2002. salvador promotes both cell cycle exit and apoptosis in Drosophila and is mutated in human cancer cell lines. Cell 110: 467-478.

Thompson BJ, Cohen SM. 2006. The Hippo pathway regulates the bantam microRNA to control cell proliferation and apoptosis in Drosophila. Cell 126: 767-774.

Tyler DM, Baker NE. 2007. Expanded and Fat regulate growth and differentiation in the Drosophila eye through multiple signaling pathways. Dev Biol 305: 187-201.

Udan RS, Kango-Singh M, Nolo R, Tao C, Halder G. 2003. Hippo promotes proliferation arrest and apoptosis in the Salvador/ Warts pathway. Nat Cell Biol 5: 914-920.
Vassilev A, Kaneko KJ, Shu H, Zhao Y, DePamphilis ML. 2001. TEAD/TEF transcription factors utilize the activation domain of YAP65, a Src/Yes-associated protein localized in the cytoplasm. Genes \& Dev 15: 1229-1241.

Wei X, Shimizu T, Lai ZC. 2007. Mob as tumor suppressor is activated by Hippo kinase for growth inhibition in Drosophila. EMBO J 26: 1772-1781.

Willecke M, Hamaratoglu F, Kango-Singh M, Udan R, Chen CL, Tao C, Zhang X, Halder G. 2006. The fat cadherin acts through the hippo tumor-suppressor pathway to regulate tissue size. Curr Biol 16: 2090-2100.

Wu S, Huang J, Dong J, Pan D. 2003. hippo encodes a Ste-20 family protein kinase that restricts cell proliferation and promotes apoptosis in conjunction with salvador and warts. Cell 114: 445-456.

Wu S, Liu Y, Zheng Y, Dong J, Pan D. 2008. The TEAD/TEF family protein Scalloped mediates transcriptional output of the Hippo growth-regulatory pathway. Dev Cell 14: 388-398.

Xu T, Wang W, Zhang S, Stewart RA, Yu W. 1995. Identifying tumor suppressors in genetic mosaics: The Drosophila lats gene encodes a putative protein kinase. Development 121: 1053-1063.

Zender L, Spector MS, Xue W, Flemming P, Cordon-Cardo C, Silke J, Fan ST, Luk JM, Wigler M, Hannon GJ, et al. 2006. Identification and validation of oncogenes in liver cancer using an integrative oncogenomic approach. Cell 125: 12531267.

Zeng Q, Hong W. 2008. The emerging role of the hippo pathway in cell contact inhibition, organ size control, and cancer development in mammals. Cancer Cell 13: 188-192.

Zhang L, Ren F, Zhang Q, Chen Y, Wang B, Jiang J. 2008. The TEAD/TEF family of transcription factor Scalloped mediates Hippo signaling in organ size control. Dev Cell 14: 377-387.

Zhao B, Wei X, Li W, Udan RS, Yang Q, Kim J, Xie J, Ikenoue T, Yu J, Li L, et al. 2007. Inactivation of YAP oncoprotein by the Hippo pathway is involved in cell contact inhibition and tissue growth control. Genes \& Dev 21: 2747-2761.

Zhao B, Lei QY, Guan KL. 2008a. The Hippo-YAP pathway: New connections between regulation of organ size and cancer. Curr Opin Cell Biol 20: 638-646.

Zhao B, Ye X, Yu J, Li L, Li W, Li S, Yu J, Lin JD, Wang CY, Chinnaiyan AM, et al. 2008b. TEAD mediates YAP-dependent gene induction and growth control. Genes \& Dev 22: 1962-1971. 


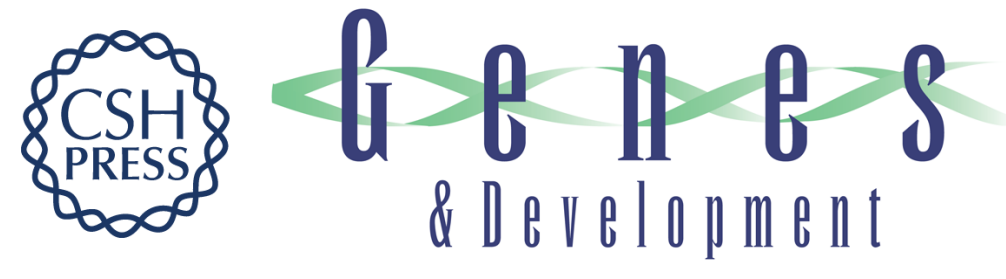

\section{Structural basis of YAP recognition by TEAD4 in the Hippo pathway}

Liming Chen, Siew Wee Chan, XiaoQian Zhang, et al.

Genes Dev. 2010, 24:

Access the most recent version at doi:10.1101/gad.1865310

Supplemental

Material

References

License

Email Alerting Service
http://genesdev.cshlp.org/content/suppl/2010/01/08/24.3.290.DC1

This article cites 58 articles, 17 of which can be accessed free at: http://genesdev.cshlp.org/content/24/3/290.full.html\#ref-list-1

Receive free email alerts when new articles cite this article - sign up in the box at the top right corner of the article or click here.

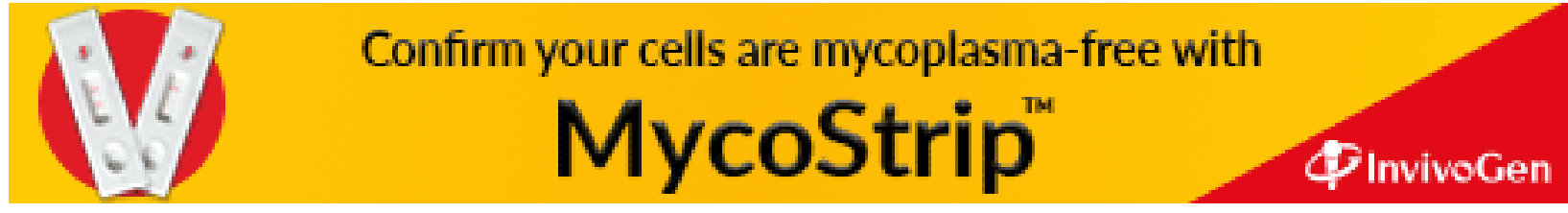

